\title{
ALIMENTOS COM AÇÃO FOTOPROTETORA: POSSÍVEL PREVENÇÃO NO CÂNCER DE PELE?
}

\section{Autores: Franciele Cristina Batista1, Vanessa Yuri Suzuki², Lorena Dal Collina Sangiuliano ${ }^{3}$, Heitor Carvalho Gomes ${ }^{4}$, Renato Santos de Oliveira Filho, ${ }^{5, A}$, Carlos Rocha Oliveira ${ }^{6}$, Lydia Masako Ferreira ${ }^{7}$}

${ }^{1}$ Aluna do Curso de Mestrado Profissional em Ciência, Tecnologia e Gestão aplicadas à Regeneração Tecidual da Unifesp. ${ }^{2}$ Profa. Coorientadora do Curso de Mestrado Profissional em Ciência, Tecnologia e Gestão aplicadas à Regeneração Tecidual da Unifesp.

${ }^{3}$ Aluna de Iniciação Científica, Graduação em Medicina pela Universidade Federal de São Paulo.

${ }^{4}$ Prof. orientador do Curso de Mestrado Profissional em Ciência, Tecnologia e Gestão aplicadas à Regeneração Tecidual da Unifesp.

${ }^{5, A}$ Prof.Afiliado e Prof. Orientador do Curso de Mestrado Profissional em Ciência, Tecnologia e Gestão aplicadas à Regeneração Tecidual da Unifesp.

${ }^{6}$ Prof. da Universidade Anhembi Morumbi. Doutor em Biologia Molecular pela Universidade Federal de São Paulo.

${ }^{7}$ Profa. Titular da Disciplina Cirurgia Plástica da Unifesp. Coordenadora do Programa Pós-graduação em Cirurgia Translacional da Unifesp. Pesquisadora CNPq $1 \mathrm{~A}$.

\section{Informações do artigo}

Palavras Chave: neoplasias cutâneas, dieta, fotoproteção.

\section{Resumo}

O câncer de pele é o mais frequente no Brasil e representa 30\% de todos os tumores malignos registrados no país. Alguns compostos bioativos presentes nos alimentos como carotenoides (betacaroteno, licopeno), probióticos e ômega-3, extrato de própolis, podem auxiliar na proteção da pele contra os danos causados por raios ultravioleta. Para atingir o objetivo foi realizada uma revisão bibliográfica para avaliar a ação fotoprotetora dos compostos bioativos, nas principais bases de dados em saúde Pubmed, Lilacs e SciElO, com palavras-chave: neoplasias cutâneas, dieta, fotoproteção, nos idiomas português e inglês, considerando o período de 2008 a 2019. O uso de betacaroteno na fotoproteção mostrou-se eficaz, com suplementação diária entre $15 \mathrm{mg}$ e $180 \mathrm{mg}$ e protegeu a pele contra queimaduras solares. Extrato de tomate contendo licopeno conferiu proteção contra danos agudos à pele causados pela exposição solar à longo prazo. A suplementação de licopeno e luteína protegeu a pele contra danos à pele causados pela radiação solar.

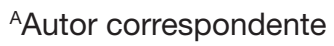

Renato Santos de Oliveira Filho - E-mail: renato.filho2@einstein.br - ORCID: https://orcid.org/0000-0002-7464-973X
}

DOI: https://doi.org/10.31415/bjns.v3i1.87 - Artigo recebido em: 10 de fevereiro de 2020 ; aceito em27 de fevereiro de 2020; publicado em 10 de março de 2020. Brazilian Journal of Natural Sciences, Vol. 3, N.1, março 2020. Disponível online a partir de 10 de março de 2020, ISSN 2595-0584. www.bjns.com.br. Todos os autores contribuíram igualmente com o artigo. Os autores declaram não haver conflito de interesse. Este é um artigo de acesso aberto sob a licença CC - BY: http://creativecommons.org/licenses/by/4.0 
A ingestão diária da bebida polifenol chá verde por 12 semanas apresentou efeito fotoprotetor. Observou-se que substâncias não tóxicas consumidas na dieta como polifenóis em frutas, vegetais, vinho, chá e cafeína, ou disponíveis na forma oral de suplementos, podem aumentar a produção de ATP e inibir a produção de ROS ou diminui a produção de PGE2, causando redução dos danos induzidos pela luz solar em humanos e protegendo a pele por vários mecanismos potenciais. Extrato de própolis tem sido utilizado no preparo de alimentos e bebidas com a finalidade de melhorar ou prevenir o câncer, e a fruta romã pode exercer ação anti-inflamatória, antiproliferativa e ter efeitos anti-tumorigênicos. Conforme os artigos estudados, o consumo dos compostos bioativos carotenoides como betacaroteno e licopeno, flavonóides, polifenóis, cafeína, ácidos graxos poli-insaturados ômega-3, vitamina E, e minerais como o selênio, são estratégias eficientes na proteção da pele contra a radiação solar, diminuindo o risco do câncer de pele. Entretanto, mais estudos clínicos randomizados precisam ser realizados para confirmar seus efeitos e estabelecer doses adequadas para efeito fotoprotetor.

\section{Article ID}

Keywords:

skin cancer, diet, photoprotection.

\section{Abstract}

Skin cancer is the most common in Brazil and represents $30 \%$ of all malignant tumors registered in the country. Some bioactive compounds present foods such as carotenoids (beta carotene, lycopene), probiotics and omega-3 propolis extract, can help protect the skin from damage caused by ultraviolet rays. To achieve the objective, a literature review was performed to evaluate a photoprotective action of bioactive compounds in the main health databases Pubmed, Lilacs and SciELO, with keywords: skin cancer, diet, photoprotection, in Portuguese and English, considering the period from 2008 to 2019. The use of beta-carotene in photoprotection was effective, with daily supplementation between $15 \mathrm{mg}$ and $180 \mathrm{mg}$ daily and protects the skin against sunburn. Included lycopene tomato extract provides protection against acute skin damage caused by long-term sun exposure. Lycopene and lutein supplementation protected the skin from damage to the skin caused by sun damage. A daily intake of polyphenol green tea drink for 12 weeks shows photoprotective effect. Note that non-toxic substances that consume diet such as polyphenols in fruits, vegetables, wine, tea and coffee, or make oral supplements available, may increase ATP production and inhibit ROS production or decrease PGE2 production, causing harm reduction. induced by sunlight in humans and protect the skin by various mechanisms used. Propolis extract was used for the preparation of food and beverages to improve or prevent cancer, and pomegranate fruit can exert anti-inflammatory, antiproliferative action and anti-tumor effects. According to the articles studied, the consumption of carotenoid bioactive compounds such as beta-carotene and lycopene, flavonoids, polyphenols, caffeine, omega-3 polyunsaturated fatty acids, vitamins and minerals such as selenium, are effective in protecting the skin against sun pollution, improving or prognosis of skin cancer. However, further randomized clinical trials need to be performed to confirm their effects and to define doses used for the photoprotective effect. 


\section{Introdução}

O câncer de pele é o mais frequente no Brasil e representa $30 \%$ de todos os tumores malignos registrados no país, segundo o Instituto Nacional de Câncer José de Alencar Gomes da Silva (INCA 2018).

O câncer de pele não melanoma é a neoplasia maligna de maior incidência no Brasil, segundo dados epidemiológicos nacionais, sendo considerado um grave problema de saúde pública, pois pode levar a deformidades físicas e ulcerações graves, onerando os custos dos serviços de saúde. Nos Estados Unidos, a incidência de câncer de pele tem aumentado exponencialmente, chegando a uma média de 900.000 a 1.200.000 casos/ano, elevando significativamente os custos das seguradoras de saúde (FERREIRA et al., 2011).

A radiação solar é considerada o fator ambiental mais importante no desenvolvimento do câncer de pele. Os estudos apontam que $80 \%$ de toda a exposição solar em nossa vida até os 18 anos de idade, e a experiência médica mostra que as pessoas que manifestam câncer de pele foram expostas a grandes quantidades de sol ao longo da vida ou receberam picos intensos dessa exposição, com queimadura solar. A radiação solar é capaz de provocar mutações e alterações nas células, e essas alterações podem ser transmitidas para as células novas resultantes do processo de multiplicação celular. Com o tempo, elas podem acumular outras mutações causando câncer de pele (OLIVEIRA et al., 2018).

A alimentação é um fator responsável por manter o sistema imunológico funcionando adequadamente, o que auxilia na prevenção do surgimento do câncer de pele. Por esse motivo torna-se relevante uma alimentação balanceada, regular e variada, rica em verduras, legumes e frutas, optando por produtos naturais e evitando os processados industrialmente, que contêm corantes e conservantes. (OLIVEIRA et al., 2018).

A nutrição pode auxiliar como fator de prevenção primária do câncer, através da ingestão de alimentos que atuam como fator de proteção contra esta doença, e evitando-se a ingestão de alimentos que contenham fatores cancerígenos. (GOMES et al., 2010)

O consumo de frutas, verduras e hortaliças, que apresentam agentes naturais com potencial antioxidante, anti-inflamatório, antimutagênico, anticarcinogênico e propriedades imunomodulatórias, demonstra surpreendentes efeitos inibitórios em di- versos processos moleculares e celulares e, portanto, atualmente ganha destaque nos estudos de prevenção de danos cutâneos causados por radiações UV (FERNÁNDEZ-GARCÍA, 2003; 2014; BETTA et al., 2018).

Existem alguns alimentos, nutrientes e compostos bioativos de alimentos (CBAs) como carotenoides, flavonoides, polifenóis, cafeína, ácidos graxos poli-insaturados ômega-3, vitamina C, vitamina E, e o selênio, que podem apresentar capacidade fotoprotetora da pele através de seu consumo regular (BOELSMA, HENDRIKS, ROZA, 2001; STAHL, SIES, 2007, 2012; ANUNCIATO, ROCHA FILHO, 2012).

Considerando o acentuado impacto do consumo de fotoprotetores orais na prevenção e tratamento do câncer de pele, torna-se relevante avaliar o impacto de nutrientes e compostos bioativos contra a radiação ultravioleta.

\section{Material e Método}

Foi realizada uma revisão bibliográfica nos periódicos disponíveis nas principais bases de dados em saúde Pubmed, Lilacs e SciElO, utilizando as palavras-chave: neoplasias cutâneas, câncer de pele, dieta, fotoproteção, vitaminas, minerais, ômega-3 nos idiomas português e inglês, considerando o período de 2008 a 2019. Foram considerados como critérios de inclusão, estudos que avaliaram o consumo e a suplementação oral de alimentos com ação fotoprotetora em pacientes adultos e idosos.

\section{Resultados}

FORTES et al. (2018), em estudo de caso-controle em hospital em Roma, avaliaram 304 pacientes recém-diagnosticados com melanoma e 305 pacientes controle responderam questionário de frequência alimentar. Após o controle de vários fatores, como histórico médico, exposição ao sol e pigmentação da pele, mostraram que aqueles que consumiram dieta típica do Mediterrâneo tiveram menor incidência de melanoma. O consumo de frutas, legumes, chá, peixe, marisco e peixe rico em ácidos graxos ômega-3 foi encontrado para ter efeito protetor. Peixes ricos em ácidos graxos ômega-3 incluem sardinha, anchova, atum e salmão. O consumo de vegetais cozidos (mais de cinco vezes por semana), vegetais crucíferos (mais de três vezes por semana), vegetais de folhas verdes (três vezes por semana), frutas (mais de uma vez por 
dia), frutas cítricas (mais de cinco vezes) por semana), e o uso de ervas frescas (quatro ou mais regularmente) foi significativamente associado a diminuição do risco de melanoma.

KOPCKE, KRUTMANN (2008), em meta-análise realizada com base em sete estudos, avaliaram a eficácia do uso de betacaroteno na fotoproteção em humanos com suplementação diária entre 15 mg e 180 mg, e demonstraram que a suplementação deste carotenoide protegeu a pele contra queimaduras solares e que esta proteção foi dependente do tempo de duração da intervenção. O tempo mínimo de suplementação para alcance da proteção foi de dez semanas, com aumento médio do efeito protetor de 0,5 desvio padrão para cada mês adicional de suplementação. A dose ideal para fotoproteção mesmo sendo controversa, apresentou efeito fotoprotetor com doses diárias a partir de $24 \mathrm{mg}$.

RIZWAN et al. (2011), em estudo clínico, avaliaram vinte mulheres que consumiram $55 \mathrm{~g}$ de extrato de tomate por dia, correspondente a $16 \mathrm{mg}$ de licopeno, acompanhado de $10 \mathrm{~g}$ de azeite de oliva, por 12 doze semanas. Os autores demonstraram que extrato de tomate contendo licopeno conferiu proteção contra danos agudos à pele causados pela exposição solar e potencialmente, também a longo prazo.

GAMBA et al. (2013), para investigar o aconselhamento nutricional sobre os efeitos de dieta com baixo teor de gordura no risco de câncer de pele, avaliaram mais de 48.000 mulheres na pós-menopausa, randomizadas para controle ou grupo de intervenção dietética. A intervenção dietética incluiu várias sessões educacionais nas quais nutricionistas instruíram os participantes sobre como diminuir o consumo total de gordura para menos de $20 \%$ do total de calorias, consumir cinco ou mais porções diárias de vegetais e consumir seis ou mais porções diárias de grãos. Após oito anos de acompanhamento, não houve diferenças na incidência de melanoma ou câncer de pele não melanoma entre os dois grupos de mulheres. Mesmo após a análise de sensibilidade, em que os participantes que não cumpriram totalmente os requisitos do estudo foram excluídos, ainda não houve associação entre a intervenção com baixo teor de gordura e o risco de câncer de pele.

FARRAR et al. (2015), em estudo clínico randomizado duplo-cego controlado por placebo 50 adultos com idade entre 18 a 65 anos e fototipo de pele I e II, consumiram $1350 \mathrm{mg}$ de extrato de chá verde encap- sulado com $50 \mathrm{mg}$ de vitamina $\mathrm{C}$, duas vezes ao dia durante três meses. Os autores observaram que não houve diferença significante entre os grupos controle e placebo em relação a eritema cutâneo, infiltração de leucócitos e efeito inflamatório de UVR.

FREITAS et al. (2015), em estudo transversal, mostraram os efeitos de antioxidantes na prevenção do câncer de pele em 84 indivíduos com idade entre 38 a 84 anos. No grupo 1, $\mathrm{n}=60$, os voluntários foram diagnosticados com câncer de pele não melanoma. O grupo 2, $\mathrm{n}=24$ foi composto de indivíduos saudáveis que nunca tiveram câncer de pele. Os autores mostraram que a ingestão de concentrações dietéticas de minerais, antioxidantes, zinco, cobre e selênio no grupo 1 foi significantemente menor em comparação ao grupo 2 . A correlação entre marcadores de estresse oxidativo e concentrações dietéticas, nutrientes e antioxidantes mostrou a influência da ingestão de alimentos de vitaminas $\mathrm{A}, \mathrm{E}$ na redução do estresse oxidativo.

SOKOLOSKI, BORGES, BAGATIN (2015), em ensaio clínico randomizado, mostraram os efeitos da suplementação oral de licopeno em 20 mulheres saudáveis na faixa etária de 40 anos, durante dez semanas. No grupo (C/ GC) dez mulheres consumiram 16 mg de licopeno em cápsulas por dia. No grupo controle (T/GT) dez mulheres consumiram duas colheres de sopa de molho de tomate com uma colher de sopa de azeite, diariamente. A ingestão oral e regular de licopeno foi segura, contudo os resultados encontrados não apresentaram nenhum efeito para foto-proteção sistêmica contra radiação ultravioleta.

BECK et al. (2017), em estudo cruzado, randomizado, duplo-cego controlado por placebo, demostraram os efeitos da suplementação oral de $5 \mathrm{mg}$ licopeno e 10 mg luteína diariamente por doze semanas. No grupo de estudo licopeno, 33 indivíduos foram recrutados, sendo homens e mulheres com idade média de 40 anos. No grupo luteína, 32 indivíduos foram selecionados, sendo homens e mulheres com idade média de 47 anos. No grupo de estudo licopeno, houve redução da radiação UV. No grupo luteína, houve redução da radiação de UVA e UVB. Em ambos os grupos suplementados com licopeno e luteína a concentração de carotenoides aumentou significantemente. Os autores observaram que a suplementação de licopeno e luteína pode proteger contra danos à pele causados pela radiação solar.

HEINRICH et al. (2011), em estudo clínico rando- 
mizado duplo-cego, controlado por placebo, mostraram os efeitos de polifenóis de chá verde em $60 \mathrm{mu}$ lheres durante 12 semanas. No grupo GT (tratado) as participantes consumiram um litro de chá verde / $1402 \mathrm{mg}$ de catequinas, diariamente. No grupo C (placebo) consumiram bebida constituinte correspondente com o mesmo sabor. A fotoproteção, estrutura e função da pele foram medidas em linha de base (semana 0 ), semana seis e semana doze. Após a exposição das áreas da pele a 1,25 dose eritérmica mínima de radiação de simulador solar, o eritema induzido por UV diminuiu significantemente no grupo de intervenção GT em 16 e $25 \%$ após seis e 12 semanas, respectivamente. Os autores mostraram que a ingestão diária da bebida polifenol chá verde por 12 semanas apresentou efeito fotoprotetor.

ANDREW et al. (2014), em revisão de literatura, observou que substâncias não tóxicas consumidas na dieta ou disponíveis na forma oral de suplementos que aumentam a produção de ATP, inibem a produção de ROS ou diminui a produção de PGE2, pode ajudar a reduzir os danos induzidos pela luz solar em humanos e proteger a pele por vários mecanismos potenciais. Estas substâncias incluem polifenóis em frutas, vegetais, vinho, chá e cafeína.

A própolis tem sido utilizada por muitos povos no preparo de alimentos e bebidas com a finalidade de melhorar ou prevenir doenças como o diabetes mellitus, inflamações, doenças cardíacas e câncer (BURDOCK, 1998; BANKOVA et al., 1998). A partir do conhecimento empírico sobre as suas propriedades medicinais, a própolis tem sido alvo de diversos estudos científicos com a finalidade de elucidar suas propriedades químicas e biológicas, determinando assim, suas possíveis substâncias ativas e mecanismos de ação SFORCIN et al. (2005), apresentou trabalhos que têm evidenciado o potencial inibitório das própolis brasileiras e de alguns dos seus componentes sobre diversos tipos de células neoplásicas, como demonstradas por LI e colaboradores (2008). De acordo com KAMIYA e colaboradores (2012), o extrato etanólico da própolis vermelha brasileira e seu componente isolado, o ácido caféico éster fenetil é capaz de induzir apoptose em células de carcinoma mamário MCF-7. Ainda não existem estudos semelhantes que demonstrem estas atividades biológicas para a própolis G6 originada da região de Mata Atlântica, situada no Estado da Bahia, Brasil, embora a atividade antimicrobiana de uma substância isolada a partir da própolis
G6 baiana já tenha sido relatada por CASTRO e colaboradores (2009).

A Punica granatum L., uma planta da família $L y$ thraceae originária da região mediterrânea é rica em compostos fitoquímicos. Em artigo de revisão, SHARMA, MCCLEES, AFAQ (2017), observaram a fruta romã, como promissor de agente quimiopreventivo, por exercer ação anti-inflamatória, antiproliferativa e ter efeitos anti-tumorigênicos.

As berries possuem uma grande quantidade de antioxidantes e flavonóides, que possuem implicações potenciais positivas no câncer. Em estudo duplo- cego controlado por placebo os autores avaliaram o efeito fotoprotetor do suco de groselha. Os participantes consumiram $250 \mathrm{ml}$ de suco de groselha quatro vezes ao dia durante seis semanas. Contudo, os resultados obtidos não mostraram efeito protetor dos raios UVA e UVB na pele S. RAY et al, (2016).

\section{Conclusão}

De acordo com a literatura científica, a suplementação de nutrientes como frutas, legumes, chá verde, peixe rico em ácidos graxos ômega-3, betacaroteno, licopeno e luteína foram associados a ação quimiopreventiva no câncer de pele. Entretanto, mais estudos clínicos randomizados devem ser realizados para confirmar seus efeitos e estabelecer a dose adequada de cada nutriente.

\section{Referências}

ANDREW C. CHEN.; DIONA L. DAMIAN \& GARY M. HALLIDAY. Photodermatol Photoimmunol Photomed. Oral and systemic photoprotection. 2014; 30: 102-111.

ANUNCIATO T.P., DA ROCHA FILHO P.A. Carotenoids and polyphenols in nutricosmetics, nutraceuticals, and cosmeceuticals. J Cosmet Dermatol. 2012 Mar;11(1):51-4.

BECK. G.; MARINI, T.; JAENICKE, W. STAHL AND J. KRUTMANN. Molecular evidence that oral supplementation with lycopene or lutein protects human skin against ultraviolet radiation: results from a double-blinded, placebo-controlled, crossover study* British Journal of Dermatology (2017) 176, pp12311240.

BÚFALO, M.C; CANDEIAS, J.M.G; SFORCIN, J.M. In vitro cytotoxic effect of brazilian Green propo- 
lis on human layngeal epdermoid carcinoma (HEp-2) cells. Evidence-based complementary and alternative medicine, v. 6, p. 483-487, 2007

BURDOCK, G.A. Review of the biological properties and toxicity of bee propolis (propolis).Food and Chemical Toxicology, v. 36, p. 347-363, 1998.

CASTRO, M.L. et al. Bioassay guided purification of the antimicrobial fraction of a Brazilian propolis from Bahia state. BMC Complementary and Alternative Medicine, v. 30, p. 9-25, 2009.

DANIEL ARCUSCHIN DE OLIVEIRA, HEITOR CARVALHO GOMES, RENATO SANTOS DE OLIVEIRA FILHO, LYDIA MASAKO FERREIRA. Câncer de pele: Conhecer para melhor combater. Editora Senac São Paulo - São Paulo - 2018.

FARRAR, M.; NICOLAOU, A.; CLARKE, A.; MASON, S.; MASSEY, K.; DEW, T.; WATSON, T.; WILLIAMSON, G.; RHODES, L. A. randomized controlled trial of green tea catechins in protection against ultraviolet radiation-induced cutaneous inflammation. Am J Clin Nutr 2015; 102:608-15. Printed in USA

Fernández-García E. Skin protection against UV light by dietary antioxidants. Food Funct. 2014 Sep;5(9):1994-2003.

FORTES, C.; MASTROENI, S.; MELCHI, F. E. e cols. Um efeito protetor da dieta mediterrânea para o melanoma cutâneo. Int J Epidemiol. 2008; 37 (5): 1018-1029.

FREITAS, A. CASTRO, L.; AGUIAR, J,; ARAUJO, C,; VISAED, M.; TUAN, B.; PINCINATO. E.; MODEL. P. Nutrición Hospitalaria: Antioxidant capacity total in non-melanoma skin cancer and its relationship with food consumption of antioxidant nutrients. 2015;31 (4): 1682-168.

FERREIRA, F.R, NASCIMENTO, L.F.C.; ROTTA, O. Fatores de risco para câncer da pele não melanoma em Taubaté, SP: Um estudo caso-controle. Rev Assoc Med Bras. 2011; 57(4):431-437.

GAMBA, C.S.; STEFANICK, M.L.; SHIKANY, J.M. et al. Dieta com baixo teor de gordura e risco de câncer de pele: a iniciativa de saúde da mulher randomizou um ensaio controlado de modificação alimentar. Cancer Epidemiol Biomarkers Prev . 2013; 22 (9): 1509-1519.

GOMES, R.; MOURA, R.F. Nutrição e câncer. In: Antunes RCP, Perdicaris AAM. Prevenção do Cancer, Barueri - SP: Manole; 2010. P. 363-74.

HEINRICH, U.; MOORE, C.; SPIRT, S.; TRON-
NIER, H.; STAHL, W. green Tea Polyphenols Provide Photoprotection, IncreaseMicrocirculation, and Modulate Skin Properties of Women. J. Nutr. 141: 1202-1208, 2011.

Instituto Nacional do Câncer [homepage na internet]. Incidência de Câncer no Brasil. [acesso em 20 maio 2019]. Disponível em: http://www1.inca.gov.br/ estimativa/2018/sintese-de-resultados-comentarios. asp.

KOPCKE, W.; KRUTMANN, J. Protection from sunburn with beta-Carotene--a meta- analysis. Photochem Photobiol. 2008 Mar-Apr;84(2):284-8.

Pomegranate for Prevention and Treatment of Cancer: An Update. Pooja Sharma, Sarah F, McCleesFarrukh Afaq. Molecules 2017, 22(1), 177; https://doi.org/10.3390/ molecules 22010177

RIZWAN, M.; RODRIGUEZ-BLANCO I.; HARBOTTLE A.; BIRCH-MACHIN MA.; WATSON, R.E; RHODES, L.E. Tomato paste rich in lycopene protects against cutaneous photodamage in humans in vivo: a randomized controlled trial. Br J Dermatol. 2011 Jan;164(1):154-62.

SOKOLOSKI, L.; BORGES, M.; BAGATINI, E. Lycopene not in pill, nor in natura has photoprotective systemic Effect. Arch Dermatol Res, 2015. 